[Aus dem hygienischen Institut der Universität zu Halle a/S.]

(Director: Prof. C. Fraenkel.)

\title{
Ueber das Wachsthum der Tuberkelbacillen auf kartoffelhaltigen Nährböden.
}

\author{
Yon \\ Dr. Fgon Tomasczewski, \\ Assistenten an der med. Unirersitatspoliklinik zu Halle a/S.
}

Seit der grundlegenden Koch'schen Veröffentlichung haben sich die weiteren bakteriologischen Arbeiten über den Tuberkelbacillus in den auch sonst üblichen Bahnen bewegt. Die feineren morphologischen Erscheinungsformen, die Wachsthumsverhältnisse auf den verschiedenartigsten Nährböden, die Bedeutung der Zahl der Bacillen und des Alters der Cultur für den Verlauf der Infection, die Fragen der natürlichen und der erworbenen Immunität, die modernen Immunisirungsmethoden, alle diese Dinge sind auch für unseren Bacillus in mehr oder minder weitem Umfange in Angriff genommen worden. Freilich hat der Erfolg nicht immer der aufgewendeten Mühe entsprochen, und als besonderes Hinderniss für die genauere Erforschung der feineren Beziehungen zwischen dem Krankheitserreger und dem thierischen Körper ist von zahlreichen Untersuchern namentlich das eigenthümliche Vẹrhalten des Tuberkelbacillus in seinen künstlichen Culturen hervorgehoben worden. So stösst schon die Herstellung einer gleichmässigen Aufschwemmung der Stäbchen auf grosse Schwierigkeiten, die selbst durch grobe mechanische Eingriffe kaum überwunden werden können. So lässt sich die Menge der im einzelnen Falle übertragenen Bakterien nicht wie sonst mit Hülfe der bequemen und sicheren Plattenmethode ermitteln, und auch die Zählung der Keime im gefärbten Deckglaspräparat versagt, weil todte und lebende Stäbchen dem Farbstoff in der nämlichen Weise zugänglich sind. Aus 
eben diesem Grunde ist sogar ein so umständliches und subtiles Verfahren wie das neuerlich von Vagedes benutzte, dre sich abgewogener und dann fein zerriebener Quantitäten rein gezüchteter Tuberkelbacillen bediente, noch nicht ganz frei von Schwächen und liefert nur dann wenigstens vergleichbare Ergebnisse, wenn man, wie der genannte Forscher allerdings gethan, stets ganz dieselben Nährböden verwendet und die Bakterien auch unter sonst völlig übereinstimmenden Verhältnissen zur Entwickelung bringt. Für die weiteren Aufgaben, die mit der Dosirung des Infectionsstoffes, mit der Prüfung der Virulenz u.s.w. in Zusammenhang stehen, ist eben deshalb auch die Frage der Züchtung der Tuberkelbacillen von der grössten Bedeutung, und im Hinblick auf diese Sachlage habe ich daher einer Aufforderung des Hrn. Professor C. Fraenkel gern entsprochen, den Einfluss wechselnder Nährböden auf das Wachsthum der Stäbchen zum Gegenstand einer erneuten eingehenden Untersuchung zu machen.

Vergegenwärtigen wir uns zunächst mit kurzen Worten die Resultate der bisher auf diesem Gebiete ausgeführten Arbeiten:

Koch war die Isolirung der Tuberkelbacillen nach verschiedenen Misserfolgen bekanntlich auf Rinderserum gelungen, das bei $58^{\circ}$ sterilisirt und bei $65^{\circ}$ erstarrt war; bei Uebertragung einer schon entwickelten Reincultur auf Agar-Agar und Fleischpeptonbouillon hatte er nur ein geringes Wachsthum beobachtet, auf Kartoffeln dagegen jede Vermehrung vermisst. Die Anschauung, dass der Erreger der Tuberculose für sein Gedeihen auf künstlichen Nährböden an die Beschaffenheit der letzteren besonders hohe Ansprūche stelle, schien dadurch genügend gerechtfertigt und erlitt auch noch keine wesentliche Erschütterung, als Roux und Nocard im Jahre 1886 mittheilten, dass ein Zusatz von 6 bis 8 Procent Glycerin zu Blutserum und Agar-Agar das Wachsthum der Tuberkelbacillen auf diesen Nährböden in ganz erheblichem Maasse fördere und das letztgenannte Substrat seiner früheren Mängel völlig entkleide. Dagegen wurde die Sachlage schon verändert durch eine Arbeit von Pawlowski aus dem Jahre 1888. Pawlowski war es geglückt, die Tuberkelbacillen auch auf Kartoffeln zu cultiviren. Das von ihm angewendete Verfahren war das folgende: In gewöhnlicher Weise hergestellte Reagensglaskartoffeln wurden geimpft, die Röhrehen dann zugeschmolzen und in einem Brütofen bei $39^{\circ}$ C. gehalten. Zwölf Tage nach der Aussaat machte sich die Entwickelung der übertragenen Bacillen dem blossen Auge schon in Form grauweisser, etwas trocken aussehender Fleckchen bemerkbar, und nach 3 bis 4 Wochen war die Cultur auf der Höhe ihrer Entwickelung angelangt. Wurde die Obertäche der Kartoffeln vor der Impfung mit einer 5 procentigen Glycerinlösung bestrichen, so gediehen die Rasen 
etwas rascher. Den wesentlichen Grund für den positiven Ausfall seiner Versuche gegenüber den früheren Misserfolgen anderer Forscher erblickte Pawlowski in zwei Umständen, einmal in dem durch das Zuschmelzen bedingten Luftabschluss und ferner in der damit zugleich gewährleisteten Verhütung einer auch nur geringfügigen Austrocknung der Kartoffeloberfläche. Was den ersten Punkt anlangt, so zeigte Sander in seiner 1889 erschienenen Arbeit, ,über das Wachsthum der Tuberkelbacillen auf pflanzlichen Nährböden," dass der Luftabschluss im Gegentheil die Entwickelung der Tuberkelbacillen auf Kartoffeln behindere. Den günstigen Einfluss reichlicher Luftzufuhr hatte in der That schon Koch hervorgehoben und neuerdings ist diese Frage auch von $\mathrm{Obici}$ auf Grund besonderer Ermittelungen ganz in dem gleichen Sinne beantwortet worden. Im Uebrigen konnte Sander aber die Angaben Pawlowski's bestätigen und dahin erweitern, dass die Tuberkelbacillen auch auf Kartoffelbrühen ein gutes Wachsthum zeigten. Letztere wurden folgendermassen bereitet: Zunächst wurden die Kartoffeln geschält, fein zerrieben und mit der doppelten Menge Wasser etwa 12 Stunden in der Kälte stehen gelassen; dann wurde abgegossen, die erhaltene Flüssigkeit bis zur völligen Klärung gekocht und nun filtrirt. Von dem schwach sauren Filtrat wurde die Hälfte bis zur schwachen Alkalescenz mit Sodalösung neutralisirt und von den beiden so gewonnenen Flüssigkeiten, der sauren wie der neutralen, wiederum die eine Hälfte mit 5 Procent Glycerin versetzt. Auf diesen vier verschiedenen Brühen gediehen die Tuberkelbacillen, auf den mit Glycerin versetzten besser, als auf den übrigen, und was besonders hervorzuheben ist, auf den sauren mindestens ebenso gut, wie auf den alkalischen. De Schweinitz hat letztere Beobachtung bestätigen können und weiter mit Hülfe genauer Titrationen gezeigt, dass mit dem Wachsthum der Tuberkelbacillen überhaupt stets eine Säureproduction verbunden ist, und zwar bei den sauren Ausgangsculturen eine stärkere als bei den neutralen.

Eine chemische Prüfung der Sander'schen Kartoffelbrühe ergab

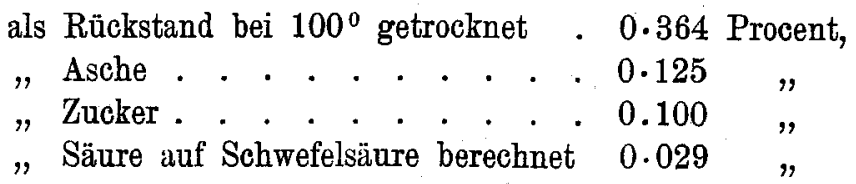

Stärke und Eiweiss waren nicht nachzuweisen. Jedenfalls war der Nährstoffgehalt dieser Kartoffelbrühe im Vergleich mit dem der bis dahin benutzten Substrate ein sehr geringfügiger.

Im Anschluss an die Sander'sche Arbeit veröffentlichte dann 1896 Lubinski Untersuchungen über das Verhalten der Tuberkelbacillen auf 
Nährmedien, die aus einem Gemisch von Glycerinagar bezw. Glycerin bouillon and der eben besprochenen Kartoffelbrühe bestanden. Die Entwickelung der Tuberkelbacillen auf diesen zusammengesetzten Nährböden begann früher und war auch weiterhin üppiger als auf Glycerinagar und Glycerinbouillon.

Lehrte uns schon das Wachsthum der Tuberkelbacillen auf der einfachen Kartoffelbrühe zur Genüge, dass man diese Mikroorganismen gewiss nicht mehr als besonders anspruchsvoll bezeichnen darf, so wurde dies durch die Forschungen der nächsten Jahre doch noch in geradezu überraschendem Maasse bestätigt. Die Nährböden, die hierbei zur Anwendung gelangten, unterschieden sich von den früher benutzten namentlich insofern, als sie nicht aus natürlichen Substanzen von thierischer oder pflanzlicher Herkunft und meist unbekannter und wechselnder Zusammensetzung aufgebaut waren, sondern ausschliesslich oder vorzugsweise bestimmte chemische Verbindungen von fester und genau ermittelter Beschaffenheit, an Stelle der Eiweiss- und Leimstoffe also besonders deren Spaltungsproducte, wie das Asparagin, das Leucin und das Tyrosin, enthielten. Nachdem schon vor langer Zeit $\mathrm{Nägeli}$ und Pasteur diesen Weg beschritten, war dann Uschinsky im Jahre 1893 wieder zu denselben zurückgekehrt und hatte in solchen Flüssigkeiten eine grosse Anzahl verschiedener Mikroorganismen zur Entwickelung bringen lrönnen. Auf den Tuberkelbacillus dehnte das gleiche Verfahren zuerst Kühne aus und benutzte anfänglich eine Lösung, die in $100^{\mathrm{ccm}}$ enthielt:

$$
\begin{array}{ll}
4 \cdot 0 \mathrm{grm} \text { Glycerin } & 0.2 \mathrm{grm} \text { Asparagin } \\
0.5 \Rightarrow \mathrm{NaCl} & 0.3 \mathrm{n} \text { schleimsaures Ammonium } \\
0.1 " \text { Tyrosin } & 0.05 " \text { Taurin. } \\
0.4 " \text { Leucin } &
\end{array}
$$

1.2 grm Aschenersatz, d. h. eine Mischung von 16.6 Chlornatrium, $3 \cdot 5^{\mathrm{grm}} \mathrm{krystallinischem} \mathrm{Magnesiumsulfat,} 1.5 \mathrm{grm}$ gebranntem Gyps, $2.5 \mathrm{grm}$ gebrannter Magnesia, 62.13 grm gebrannter Pottasche, 7.35 grm Soda, $6.2 \mathrm{grm}$ Ferrum reductum, 5.0 grm Phosphorsăure von 1.3 spez. Gewicht, 50 bis $60 \mathrm{grm}$ Milchsäure von $1.2 \mathrm{spec}$. Gewicht, aufgekocht mit 600 ocm Wasser. Diese Mischung enthielt die anorganischen Bestandtheile des Liebig'schen Fleischextractes in annähernd gleichen Mengenverhältnissen. Im Verlauf seiner Untersuchungen vereinfachte Kühne dann dieses Substrat, indem er zuerst das Taurin, später auch das Leucin und Tyrosin und endlich auch das schleimsaure Ammon in Wegfall brachte. Auf allen diesen Gemischen entwickelten sich die Tuberkelbacillen mit grosser Ueppigkeit, und erst bei weiterer Schmälerung der Kost begann das Wachsthum auszubleiben. Immer aber zeigte sich das Gly- 
cerin als ganz unentbehrlich für den Erfolg; denn sobald dasselbe fehlte, versagte auch alsbald die Entwickelung. Dass indess auch mit diesen Combinationen die untere Grenze der Ernährungsbedingungen für den Tuberkelbacillus noch nicht erreicht sei, ging aus der im gleichen Jahre erfolgten Veröffentlichung von Proskauer und Beck hervor. In Verrollständigung und weiterer Fortführung der Kühne'schen Versuche fanden die genannten Forscher, dass das in der ursprünglichen Kūhne'schen Lősung enthaltene Taurin nicht nur unnöthig, sondern sogar schädlich sei und dass unter den übrigen organischen Verbindungen dem Asparagin, namentlich aber dem Leucin eine besondere Wichtigkeit zukomme. Das letztere liess sich auch durch das Alanin und das Glycocoll ersetzen und auf 0.2 bis 0.4 procent. Lösungen aller dieser Stoffe mit 4 Procent Glycerin, 0.2 Chlornatrium und dem Kühne'schen Aschenersatz ein reichliches Wachsthum der Tuberkelbacillen erzielen. Weitere ausserordentlich eingehende und sorgfältige Ermittelungen betrafen dann die Prüfung der einzelnen Mineralsalze auf ihre etwaige Entbehrlichkeit; es zeigte sich, dass in der That die Mehrzahl der bisher benutzten anorganischen Verbindungen überfiüssig war, duss sogar das Kochsalz fortgelassen werden konnte, ohne die Nährfähigkeit der Flüssigkeit zu beeinträehtigen und dass die Anwesenheit eines Alkaliphosphates, eines Magnesiumsalzes und eines Sulfates bereits genügt, um die Entwickelung der Tuberkelbacillen zu ermöglichen. Erhöht wird die Brauchbarkeit aller derartigen Lösungen durch den Zusatz eines Kohlehydrates, wie Dextrin, Mannose, Rohrzucker u. s. w. oder.eines mehratomigen Alkoholes wie Dulcit, Mannit u. s. f., und zwar in so ausgesprochenem Maasse, dass schliesslich sogar die stickstoffhaltigen organischen Verbindungen in Fortfall kommen und dureh Salmiak oder Ammoniumsulfat ersetzt werden konnten. Schliesslich dürfen an die Stelle der Kohlehydrate bezw. der mehratomigen Alkohole unmittelbar die Weinsäure, Citronensäure, Milchsäure bezw. deren Salze treten. Endlich haben Proskauer und Beck selbst auf einem Substrat noch eine, wenn auch verzögerte Vermehrung der Tuberkelbacillen erhalten, das nur aus Glycerin 1.5, Ammoniumsulfat 0.35, Magnesiumsulfat 0.25 und primärem Kaliumphosphat 0.15 Procent bestand. Eine weitere Herabsetzung der Wachsthumsbedingungen für die Tuberkelbacillen war nicht wohl zu erwirten und kaum möglich; immerhin ist es bemerkenswerth, dass es in gleichem Jahre C. Fraenkel gelang, auf einem Nährboden noch Entwickelung zu erzielen, der der schwefelhaltigen Verbindung völlig ermangelte. Für alle diese einfachen Substrate erwies sich, wie schon für die $K u ̈ h n e$ 'schen bemerkt, nur das Glycerin als durchaus unentbehrlich. 
Ausserhalb des Rahmens der bisher erwähnten Arbeiten und auch auf nur zweifelhafter Grundlage stehen die Versuche von H. Martin und G. Marpmann. Der Erstere stellte sich Nährbouillon bezw. Nähragar aus dem Fleisch verschiedener Thiere unter Zusatz von 6 Procent Glycerin her. Es zeigte sich nun, dass das Wachsthum der Tuberkelbacillen je nach der verweudeten Thierart grosse Abweichungen aufwies. Obenan stand an Nährwerth das Heringsfleisch; diesem folgten -der Reihe nach das Fleisch der Austern, Muscheln, Affen, Pferden, Kälber, Kaninchen, Hühner, Tauben, Gänse, Hunde, Katzeń und Ratten. Ausserdem empfiehlt Martin als ein allgemeines, das Tuberkelbacillenwachsthum förderndes Mittel die Verwendung von Mineralwässern, namentlich das Wasser von Enghien und Mont-Dor bei der Bereitung der Substrate. MI arpmann behauptet die Bedeutung des glycerinphosphorsauren Kalkes als Zusatz zum Agar-Agar und will ein besonders üppiges Wachsthum auf Lecithinn ährböden beobachtet haben.

Damit wäre die Reibe der für den Tuberkelbacillus bisher benutzten Nährböden im Grossen und Ganzen erschöpft. Als wichtigstes Ergebniss aus allen diesen Untersuchungen aber kann wohl die schon vorhin vermerkte Thatsache bezeichnet werden, dass der Tuberkelbacillus auch auf sehr einfachen Substraten noch ohne Schwierigkeiten zu gedeihen vermag und die beobachtete Entwickelung, weder was Schnelligkeit noch was Ueppigkeit angeht, überhaupt deutliche Unterschiede zwischen den reichsten und den an Nähirstoffen ärmsten Medien erkennen lässt. Eine Ausnahme von dieser Regel will nur Sander festgestellt haben, der auf festen und flüssigen, glycerinhaltigen und glycerinfreien, alkalisch und sauer reagirenden Kartoffelnährböden stets eine ungemein frühe und auch in der Folge sehr starke Vermehrung erreicht zu haben angiebt. Es sei mir gestattet, den wichtigsten unter den hierauf bezüglichen Schlusssätzen aus der genannten Arbeit hervorzuheben: „Das Wachsthum auf den Kartoffeluährböden ist im Allgemeinen üppiger und geht schneller vor sich, als auf den entsprechenden thierischen; diese Eigenschaften sind bei der zweiten und dritten Generation noch ausgesprochener."

Diese Beobachtung auf ihre Richtigkeit zu prüfen und von hier aus das Verhalten der Tuberkelbacillen auf den planzlichen Substraten weiter zu verfolgen, ist im Wesentjichen das Ziel meiner eigenen Untersuchungen gewesen. Ich beimpfte zu diesem Zwecke zunächst nach der Globig'sehen Methode hergestellte Reagensglaskartoffeln mit Tuberkelbacillen, die aus jungen Glycerinagar- und Glycerinbouillonculturen eines alten; seit einiger Zeit (21/2 Jahren) im hiesigen hygienischen Institut fortgeführten Stammes von Säugethiertuberculose herrührten. Die Rōhr- 
chen wurden dann theils zugeschmolzen, theils mit Wattep fropf und Gummikappe verschlossen. Das Ergebniss war für beide Ausgangsculturen ein fast völlig negatives. Wenige Ausnahmen abgerechnet, erfolgte überhaupt kein Wachsthum, in diesen Fällen aber trat dasselbe erst nach etwa drei Wochen ein und war auch weiterhin nur ein sehr kümmerliches. Ganz dias gleiche Resultat erhielt ich dann noch in mehreren anderen in der nämlichen Weise ausgeführten Versuchen.

Die Oberfläche der Kartoffelstückchen wurde nun mit Glycerin bestrichen und darauf $m$ ist eine bald mässige, bald jedoch sehr üppige und rasche Entwickelung wahrgenommen, gleichgültig ob Glycerinagar- oder Glycerinbouillonculturen zur Aussaat benutzt, ob die Röhrchen zugeschmolzen oder nur mit Wattebausch und Gummikappe versehen worden waren. Einige Male freilich zeigten die letzteren eine etwas bessere Vermehrung, und in der Folge kam daher nur noch diese Art des Versehlusses zur Anwendung.

Die nachstehenden Tabellen veranschaulichen das Wachsthum der ersten, zweiten und dritten Generation auf festen Kartoffeln. Es bedeuten:

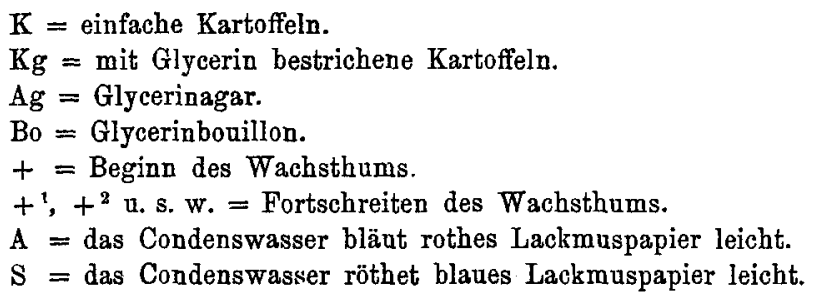

In den folgenden Tabellen mag die verschiedene Reaction der einzelnen Kartoffelröhrchen auffallen. Sie sind in gleicher Weise hergestellt, es ist dieselbe Kartoffelsorte benutzt; die Ursache muss also wohl in den Reagensröhrchen zu suchen sein.

Versuch I: Auf den einfachen Kartoffelscheiben kein Wachsthum, auf den mit Glycerin versetzten ein etwas später eintretendes als auf den Glycerinagarröhrchen; auf den letzteren das Wachsthum gleichmässiger. Auf zwei Glycerinkartoffelröhrchen sind die Impflinge überaus üppig gediehen, weit üppiger als auf sämmtlichen Glycerinagarröhrchen. Ein Einfluss der Reaction auf die Entwickelung ist nicht nachweisbar; die Reaction der einzelnen Kartoffel- und Agarröhrchen ist beim Beginn des Versuches die gleiche wie am Ende. 

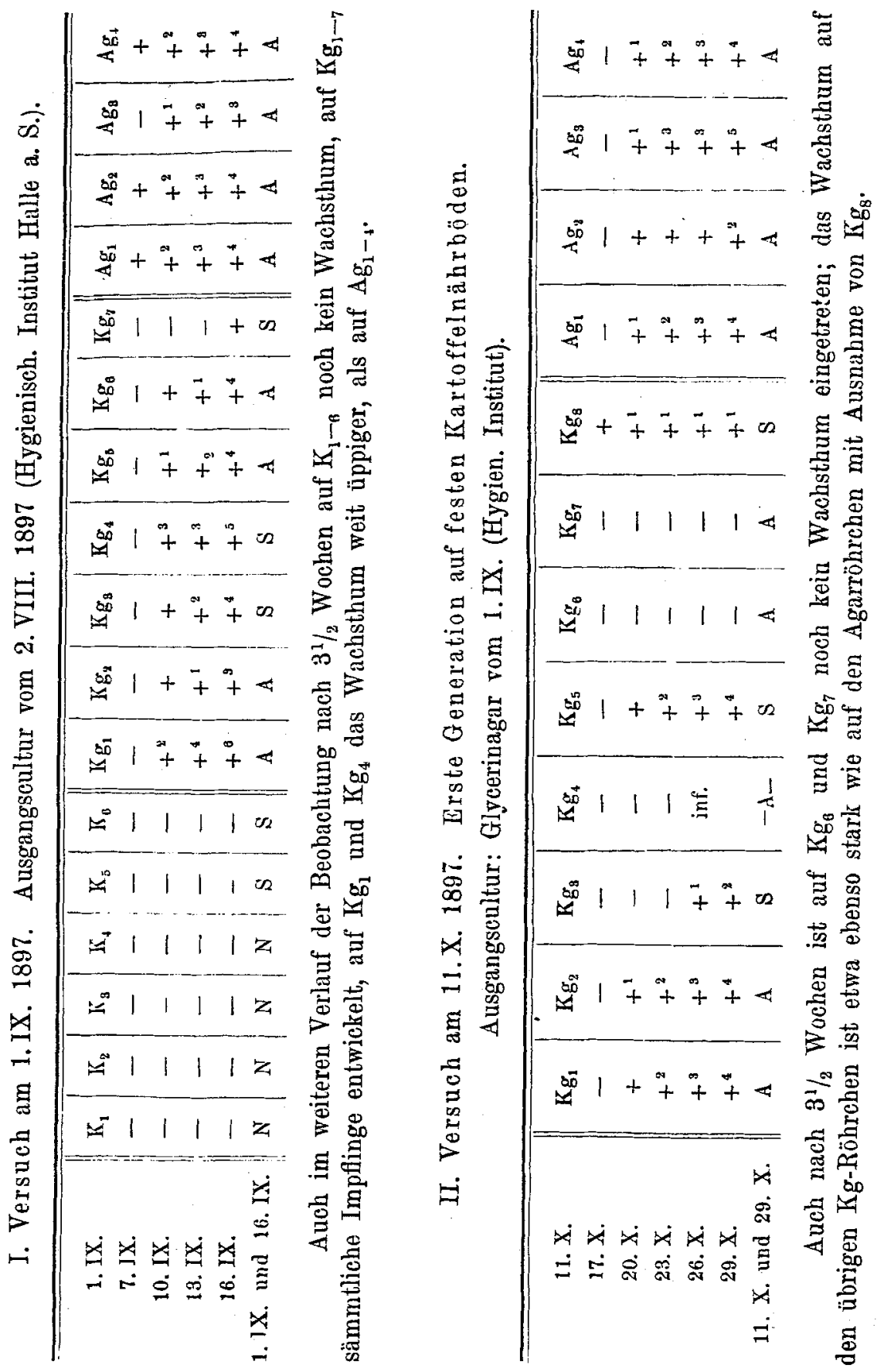


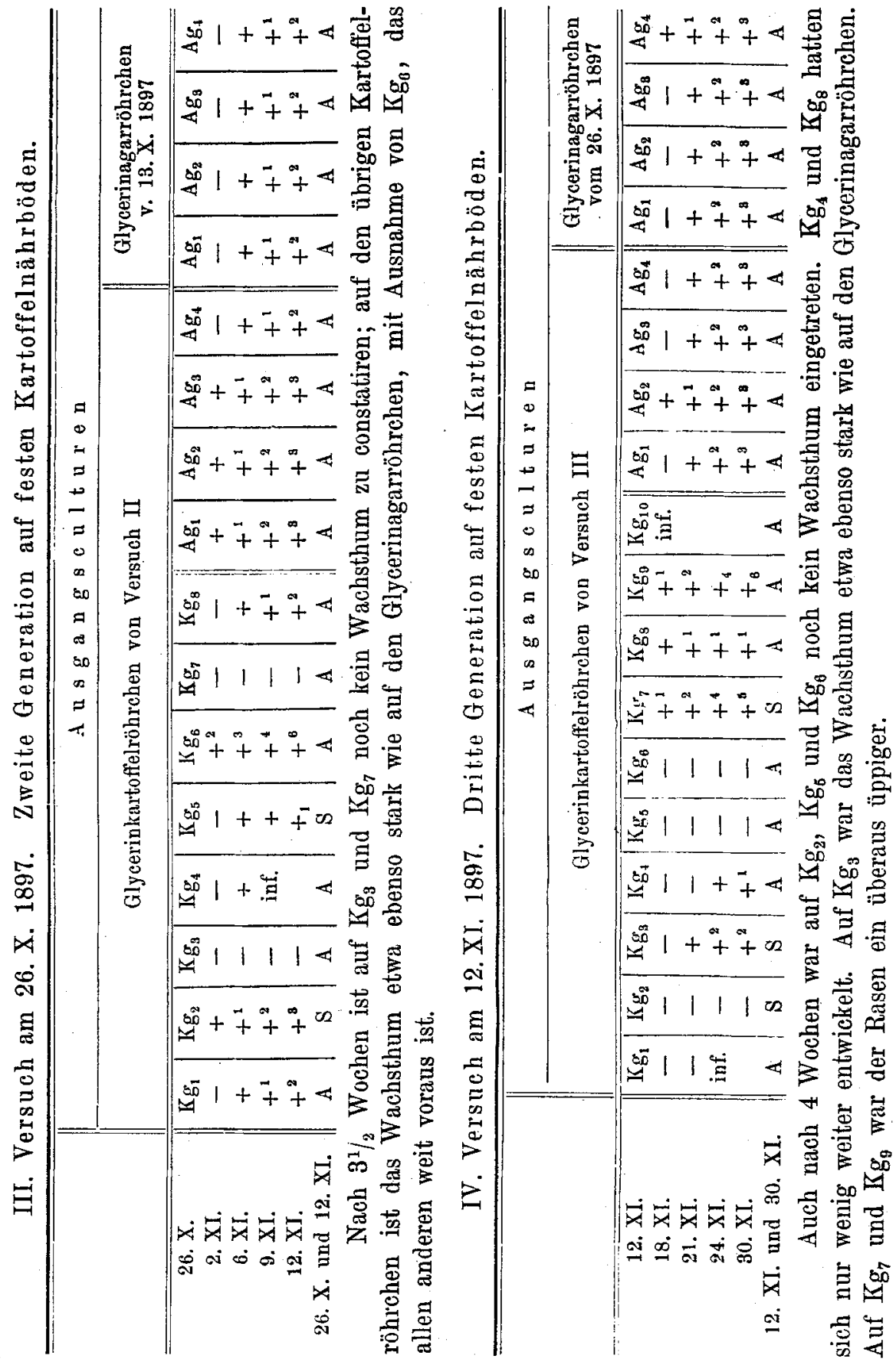


Versuch II: Die einfachen Kartoffeln wurden fortgelassen, da die Impflinge auf ihnen wiederholt nicht gediehen waren. Der Beginn der Entwickelung erfolgt auf den Glycerinkartoffelröhrchen und den Glycerinagarröhrchen meist etwa zur nämlichen Zeit; indess ist das Wachsthum auf ersteren ein viel ungleichmässigeres; anf drei Röhrchen ist es gänzlich ausgeblieben, auf einem erst spät aufgetreten. Im weiteren Verlauf ist das Wachsthum auf beiden Nährböden bei den überhaupt entwickelten Culturen ein annähernd gleich starkes. Ein Einfluss der Reaction ist nicht zu erkennen; dieselbe ist bei den einzelnen Kartoffel- und Agarröhrchen zu Beginn des Versuches die gleiche wie am Ende.

Versuch III: Das Wachsthum der zweiten Generation auf Glycerinkartoffelröhrchen tritt nicht früher ein, als auf den Glycerinagarröhrchen und ist im Ganzen ein sehr ungleichmässiges; während es auf zwei Röhrchen völlig fehlt, halten die übrigen Impflinge in ihrer Entwickelung etwa gleichen Schritt mit den Glycerinagarröhrchen; nur auf einem ist der Rasen ungemein üppig, ohne dass sich dafür eine Ursache bätte ermitteln lassen. Von den Glycerinagarröhrchen zeigen die von der Glycerinkartoffelcultur geimpften ein etwas früheres und stärkeres Wachsthum, als die von der Glycerinagarcultur geimpften. Bezüglich der Reaction gilt das für Versuch II Angegebene.

Versuch IV: Das Wachsthum der dritten Generation auf Glycerinkartoffeln setzt bei drei Röhrchen eher ein, als auf den Controlröhrchen; bei mehreren kommt es überhaupt nicht zu einer Entwickelung der Impflinge; der Rest zeigt die gleichen Verhältnisse wie auf Glycerinagar. Der frühzeitige Wachsthumsbeginn auf den erwähnten drei Röhrchen führt bei zweien zu einer sehr üppigen Wucherung auch im weiteren Verlauf. Für die Reaction des Condenswassers zu Beginn und am Ende des Versuches, sowie für die Entwickelung auf Glycerinagar gilt das im Versuch III Gesagte.

Die hiermit beschriebenen Versuchsreihen stehen nicht etwa vereinzelt da. In mehrfachen Wiederholungen kamen wohl geringe Schwankungen vor, im Wesentlichen war das Ergebniss immer das gleiche, und zwar, wie ich ausdrücklich hervorheben möchte, für vier verschiedene Stammculturen, nämlich die schon erwähnte aus dem hiesigen hygienischen Institute, eine zweite von Hrn. Prof. Wernicke in Marburg, eine dritte von Hrn. Prof. Wladimiroff in Petersburg und eine vierte von Hrn. Prof. Günther in Berlin. Den genannten drei Herren sage ich auch an dieser Stelle meinen besten Dank für ihr liebenswürdiges Entgegenkommen. Nan muss es darnach wohl als eine gesicherte Thatsache betrachten, dass für das von mir benutzte Ma- 
terial, Stammeulturen und Kartoffelsorten, die von Sander beobachteten Wachsthumsverhältnisse nicht zutreffen. Auf den einfachen Kartoffelröhrchen gelangten die Impflinge überhaupt nur ausnahmsweise zu einer geringen Vermehrung; auf den Glycerinkartoffeln war die Entwickelung weder im Allgemeinen üppiger, noch ging sie wesentlich schneller vor sich, als auf dem zur Controle geimpften Glycerinagar, und endlich trat eine Aenderung dieser Verhältnisse auch bei der $z$ weiten und dritten Generation auf diesem Nährboden nicht ein. Immerhin ist es gewiss bemerkenswerth, dass fast in jedem Versuche ein oder $\mathrm{z}$ wei Kartoffelröhrchen ein frühzeitiges und weiterhin ausserordentlich üppiges Wachsthum zeigten, während die anderen Röhrchen zum Theil steril blieben, obne dass sich für die Verschiedenheiten ein Grund ermitteln liess. Um etwaige gröbere Differenzen in der Menge der Aussaat als Ursache hierfür auszuschliessen, habe ich von einer gleichmässigen Emulsion einer jungen Bouilloncultur je zwei Oesen auf Glycerinkartoffeln überimpft; aber auch dann das gleiche Verhalten.

Im engen Anschluss an die Arbeit von Sander wurde ferner das Wachsthum der Tuberkelbacillen auf flüssigen Kartoffelnährböden, den sogenannten Kartoffelbrühen, geprüft, die in der von Sander angegebenen und schon erwähnten Weise hergestellt wurden. Je nach der Reaction und dem Glyceringehalt sind nun zu unterscheiden:

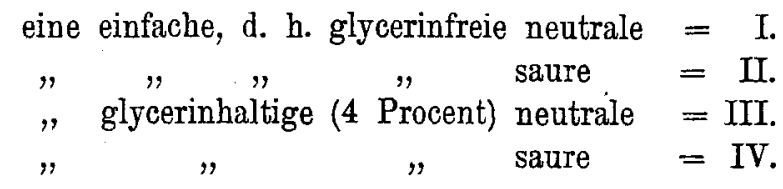

Diese Substrate wurden in Erlenmeyer'sche Kölbchen gefüllt, mit Tuberkelculturen von Glycerinbouillon und Glycerinagar geimpft und nur mit Wattepfropfen verschlossen. Zur Controle wurden Glycerinbouillonkölbchen und Glycerinagarröhrchen geimpft.

Es sei hier ausdrücklich bemerkt, dass auch für diese Untersuchungen die erwähnten vier Stämme von Tuberkelbacillen als Ausgangsculturen zur Verwendung gelangten, und für jeden einzelnen Stamm die Versuche eine mehrfache Wiederholung erfuhren. Die Ergebnisse nun waren, von geringen Schwankungen abgesehen, stets etwa die gleichen, und somit stellt die folgende Versuchsreihe gewissermassen den Typus des Wachsthums dar, das die Tuberkelbacillen auf Kartoffelbrühen zeigen. 


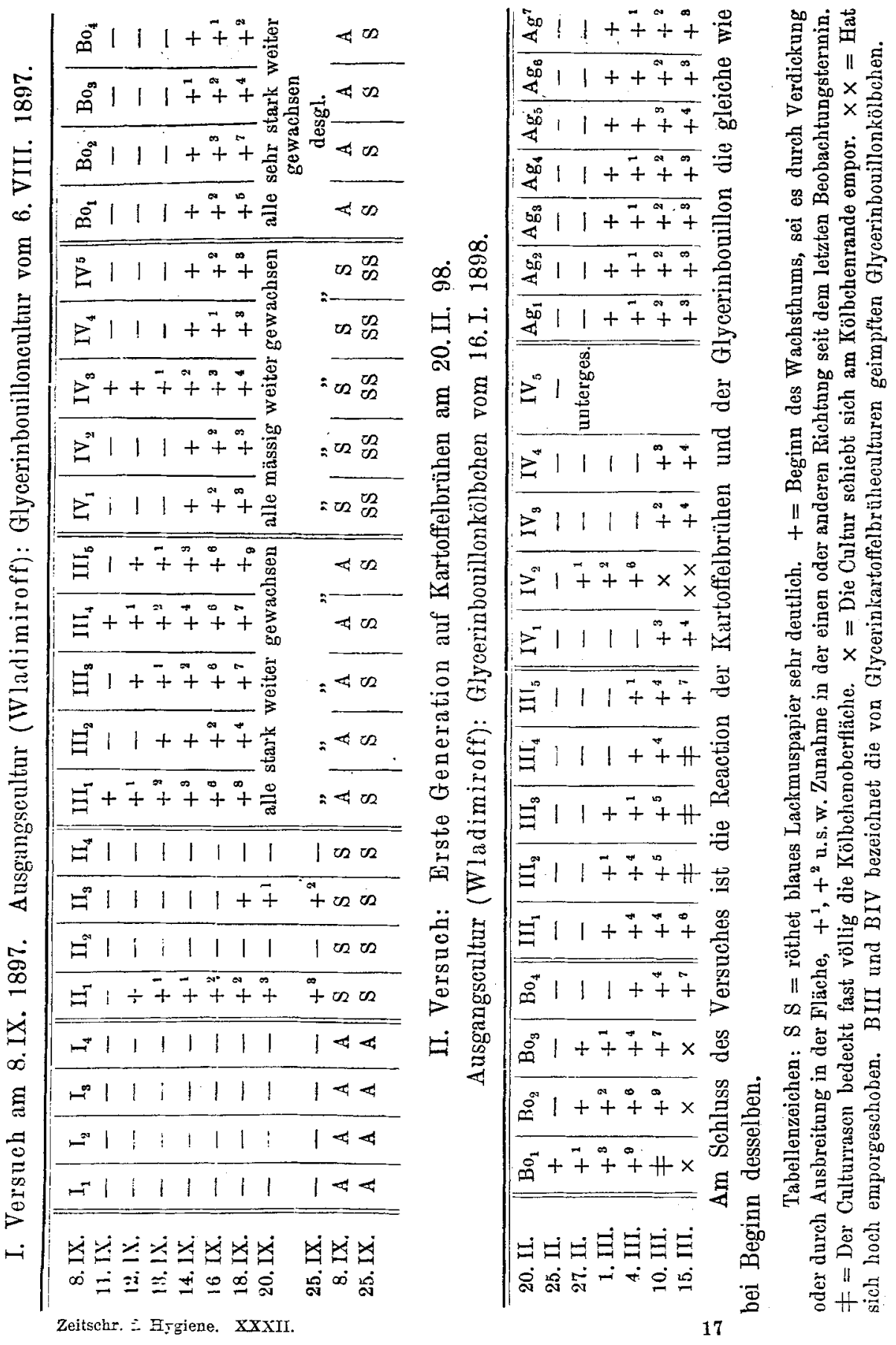




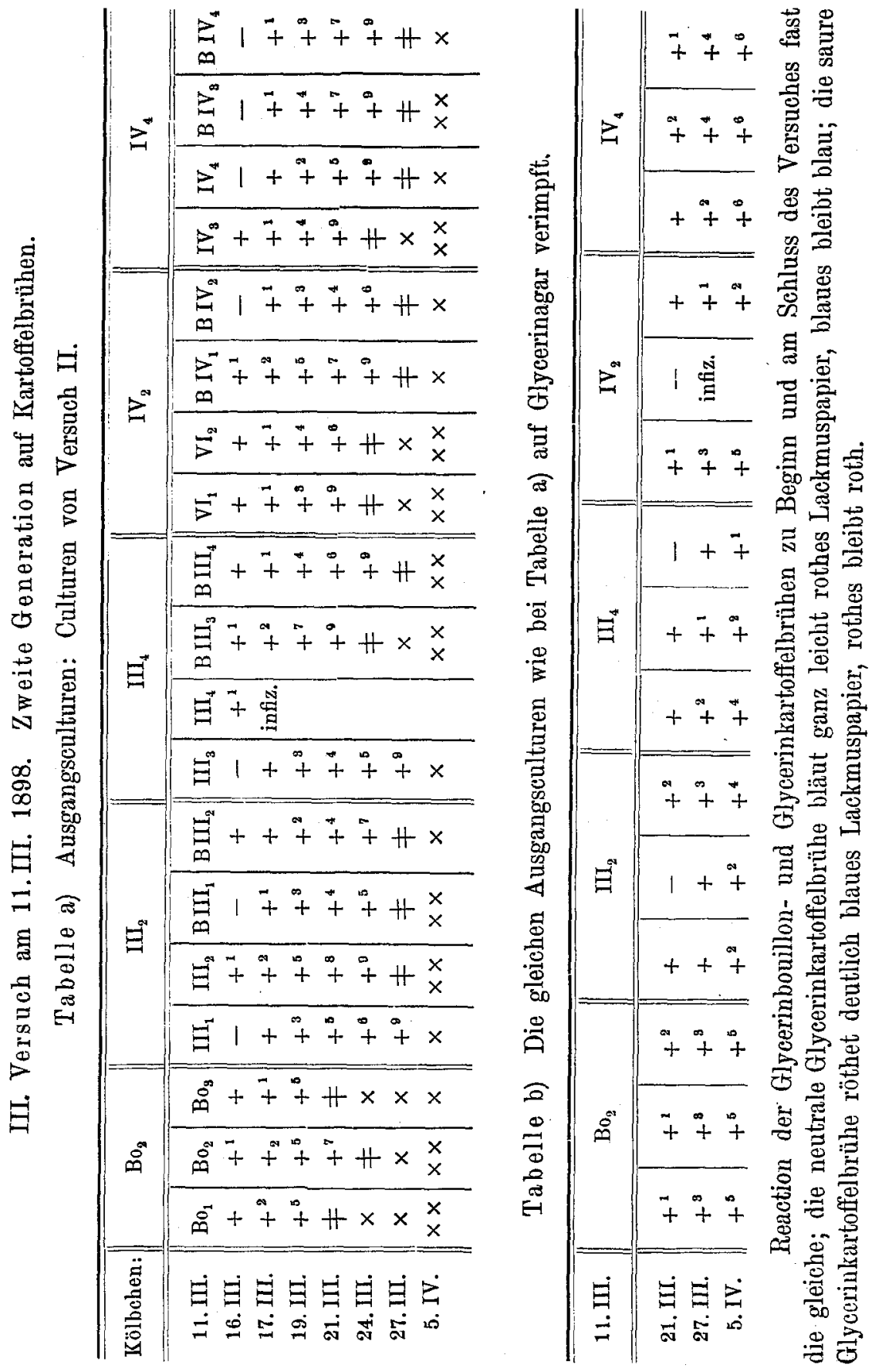


WACHSTHUM DER TUBERKELBAOILLEN.
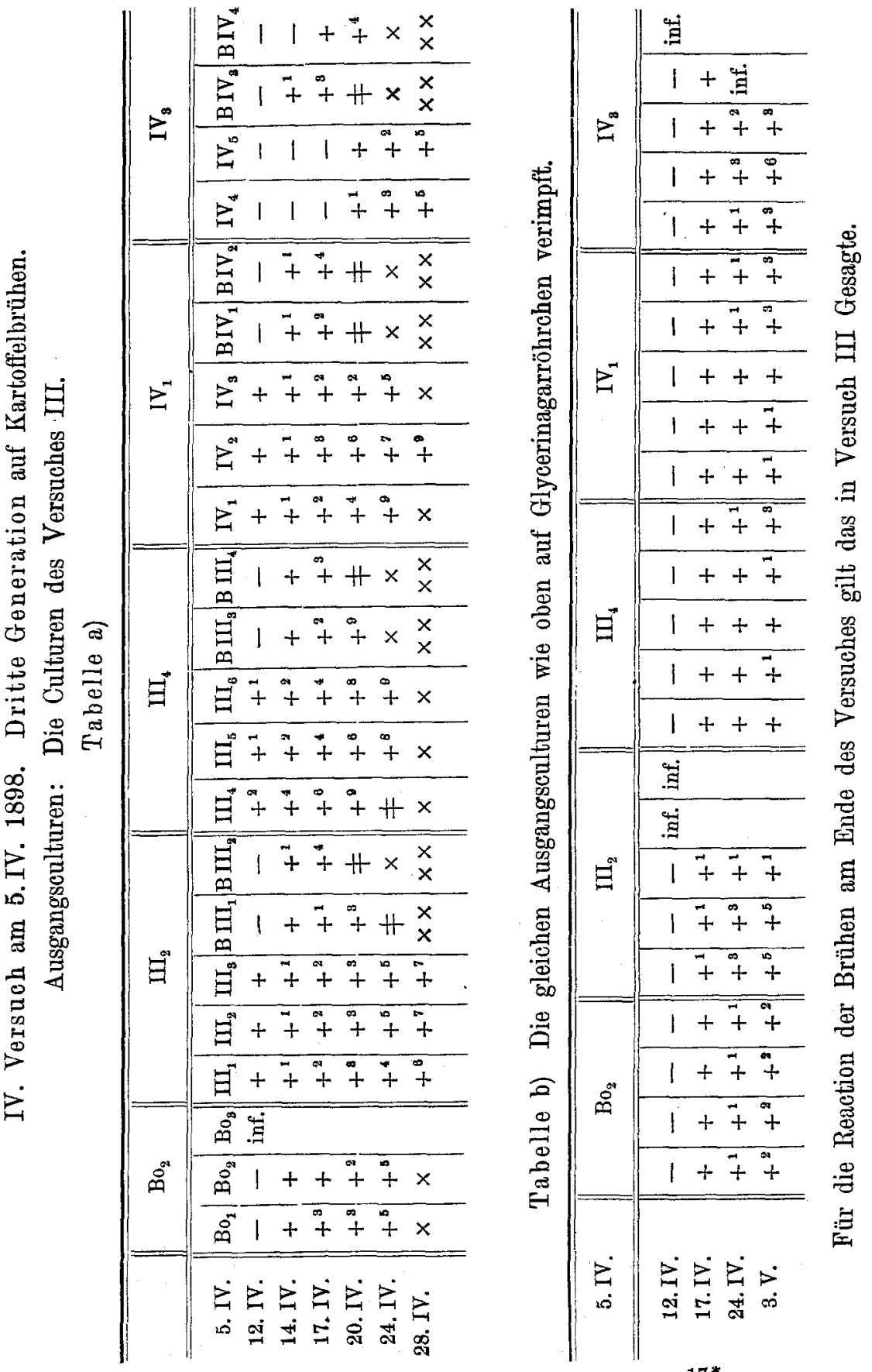

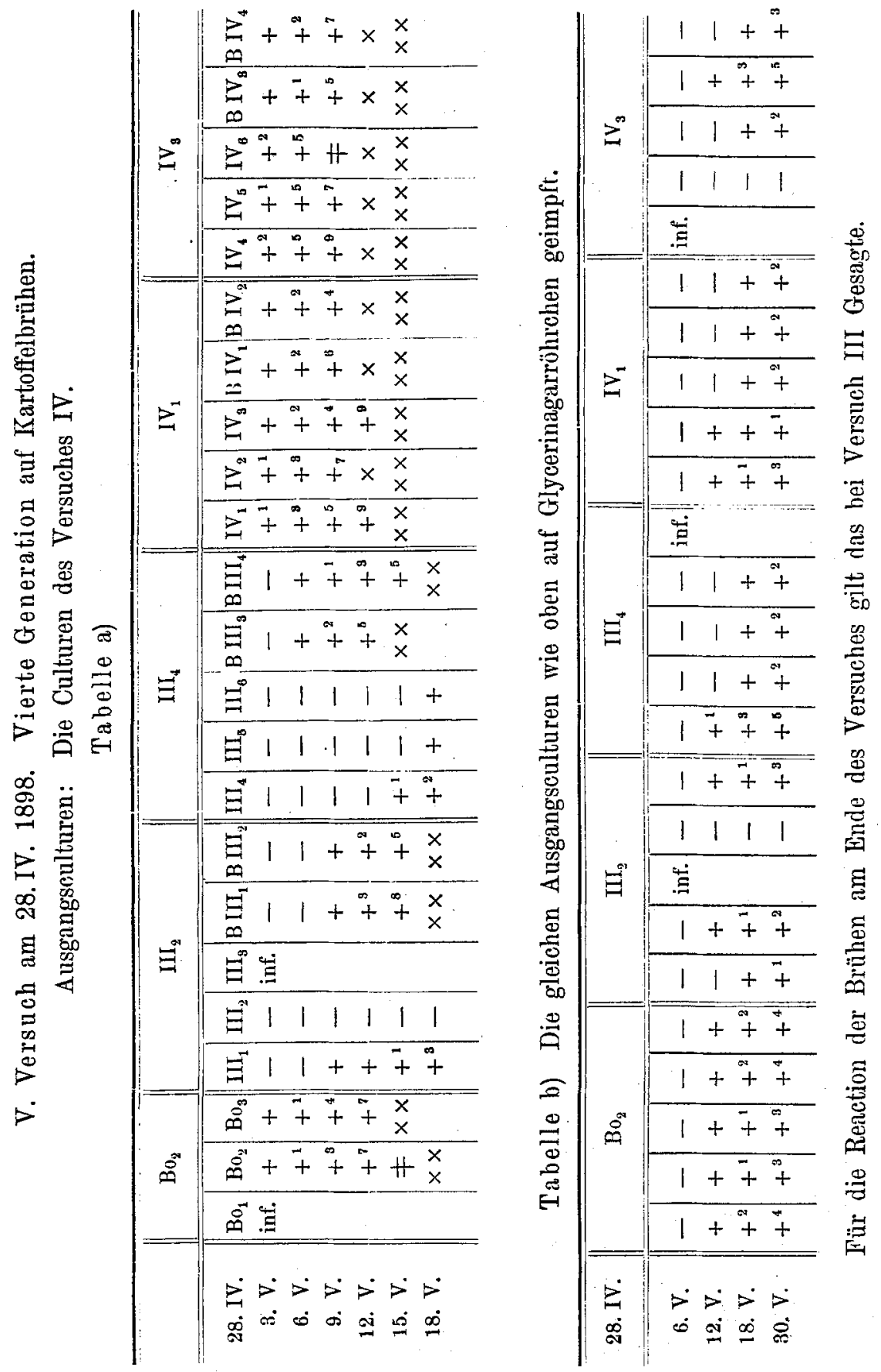
Versuch I: Das Wachsthum auf den einfachen Kartoffelbrühen, der sauren wie der neutralen, ist während der ganzen Versuchsdauer ein kaum nennenswerthes gewesen. Die Impflinge auf III hingegen begannen sich schon am dritten Tage zu entwickeln, fast 3 Tage früher als auf der sauren Glycerinkartoffelbrühe und der Glycerinbouillon. Auf letzteren beiden Nährböden fing die Entwickelung zur gleichen Zeit an, auf IV erst schwach, dann stärker; auf Glycerinbouillon war die Entwickelung, nachdem sie einmal eingesetzt, eine so reichliche, dass diese Culturen bald allen anderen weit voraus waren, und dieses Verhalten blieb auch bis zum Schluss der Beobachtung unverändert. Als am Ende des Experimentes die Reaction der Kartoffelbrühen geprüft wurde, reagirten III $_{1}$ bis III $_{4}$ schwach, III ${ }_{5}$ stark sauer, $V_{1}$ bis $\nabla_{5}$ jedenfalls saurer als zur Zeit der Impfung.

Der Versuch, von diesen Culturen eine zweite Generation auf Kartoffelbrühen zu züchten, missglückte nun wider jedes Erwarten völlig, da die Impflinge auch nicht die Spur eines Wachsthums zeigten und nach spätestens 14 Tagen untergesunken waren. Dieser Verlauf der Weiterzüchtung ist mir übrigens mehrere Nale vorgekommen und liess sich in keinem Falle auf irgend welche Besonderheit bezw. Unbrauchbarkeit der benutzten Brühen zurückführen.

Von den Brühen III und IV des Versuches I hatte ich auch Glycerinagar geimpft. Erst nach 6 bis 8 Wochen trat dann hier ein für das blosse Auge eben erkennbares Wachsthum und weiterhin das auch sonst gewöhnliche culturelle Verhalten ein.

Versuch II: Ausgangscultur: Glycerinbouillonkölbchen, geimpft am 16.I. 1898; am 20.II. 1898 sehr üppiges Wachsthum, der Rasen beginnt sich eben am Rande des Kölbchens emporzuschieben.

Es wurden geimpft:

1. fünf neutrale Glycerinkartoffellölbchen $\mathrm{III}_{1-\tilde{n}}$,

2. fünf saure Glycerinkartoffelkölbchen $I_{1-5}$,

3. vier Glycerinbouillonkölbchen $\mathrm{Bo}_{1-4}$,

4. sieben Glycerinagarröhrchen.

Reaction der Kartoffelbrühen bei Beginn des Versuches: Kartoffelbrühe IV röthet deutlich blaues Lackmuspapier, rothes bleibt roth.

Kartoffelbrühe III bläut eben rothes Lackmuspapier, blaues wird nicht geröthet.

Hinsichtlich des Wachsthumsbeginnes sind die Impflinge auf Glycerinbouillon denen auf der neutralen und sauren Glycerinkartoffelbrühe etwas voraus; auch weiterhin ist das Wachsthum der ersteren ein schnelleres und kräftigeres. Ungeachtet dieser Unterschiede sinken die Culturen auf 
den Glycerinkartoffelbrühen früher unter als diejenigen auf der Glycerinbouillon. Im Aussehen ist eine erhebliche Abweichung zwischen den Culturen auf pflanzlichen und denen auf thierischen Nährböden nicht vorhanden. Bei beiden bleibt das Nährsubstrat vollkommen klar, aber während die Culturen auf Glycerinbouillon überall gleichmässig dick und gefaltet erscheinen, zeigen diejenigen auf den Glycerinkartoffelbrühen für gewöhnlich einen gewissen Gegensatz zwischen Mitte und Randbezirk. Anfangs nämlich verdicken sich die Impflinge auf diesen Brühen stark und wachsen so bis etwa zu Zweimarkstückgrösse heran; dann aber geht von der so gestalteten Cultur weiterhin sehr häufig eine ganz feine, blaugraue, scheinbar völlig glatte Haut aus.

Versuch III: Zweite Generation auf den Kartoffelbrühen am 11.III. 98:

Ausgangsculturen: Die Culturen des vorigen Versuches, und zwar auf Glycerinbouillon, neutraler und saurer Glycerinkartoffelbrühe.

Es wurden geimpft:

1. von Glycerinbouilloncultur drei Glycerinbouillonkölbchen und drei Glycerinagarröhrchen;

2. von Cultur auf neutraler Glycerinkartoffelbrühe vier Kölbchen mit gleicher Brühe, vier Kölbchen mit Glycerinbouillon und sechs Glycerinagarröhrchen;

3. von Cultur auf saurer Glycerinkartoffelbrühe fünf Kölbchen mit gleicher Brühe, vier Kölbchen mit Glycerinbouillon und sechs Glycerinagarröhrchen.

Es ist keine Beschleunigung des Wachsthums der zweiten Generation auf den Kartoffelbrühen eingetreten. Der Entwickelungsbeginn liegt bei allen Kölbchen um den 5. bezw. 6. Tag nach der Impfung herum, und auch der weitere Verlauf weist keine nennenswerthen Unterschiede auf: indess ist der Culturrasen auch hier wieder auf allen Glycerinbouillonkölbchen ein gleichmässiger, während bei der Mehrzahl der Glycerinkartoffelbrühen von dem anfänglich vergrösserten und verdickten Impfstück theils ein fädiges Gitterwerk, theils eine sehr feine, fast ganz glatte, blaugrau erscheinende Haut ausstrahlt. Die Culturen auf den Glycerinkartoffelbrühen sinken ebenfalls wieder früher unter als diejenigen auf Glycerinbouillon. Was die Glycerinagarröhrchen anlangt, so wurden dieselben aus zwei Gründen geimpft. Einmal konnte so auch bei eventuellem Untersirken des Impfmaterials auf den Brühen die Lebensfähigkeit der Ausgangsculturen controlirt werden, und dann war ja immerhin ein Unterschied im Wachsthumsverlauf auf festen Nährböden bei der Differenz der Ausgangseulturen möglich. Da beides nicht der Fall, ist eine Erörterung der Tabelle IIIb überflüssig. 
Versuch IV: Dritte Generation auf den Kartoffelbrühen am 5. IV. 98.

Hinsichtlich der Ausgangsculturen und der von diesen geimpften Nährböden gilt cet. par. das für Versuch III Gesagte.

Das Wachsthum der dritten Generation auf den Glycerinkartoffelbrühen hat durchschnittlich etwas früher als auf der Glycerinbouillon eingesetzt; im weiteren Verlauf ist kein merklicher Unterschied zu Gunsten der Glycerinkartoffelbrühen zu bemerken; hingegen sind die von Glycerinkartoffelbrühen geimpften Glycerinbouillonkölbchen den von derselben Ausgangscultur geimpften Glycerinkartoffelbrühen etwas voraus. Für alle übrigen Punkte gilt das bei Versuch II und III Angegebene. brühen.

Versuch V: Am 28. IV. 1898 vierte Generation auf den Kartoffel-

Ausgangsculturen und Nährböden cet. par. die nämlichen wie in Versuch III.

In diesem Versuch ist die sehr späte Entwickelung der Impflinge auf der neutralen, das frühzeitige und kräftige Wachsthum derselben auf der sauren Glycerinkartoffelbrühe hervorzuheben. Indess hält im weiteren Verlauf das Wachsthum der Glycerinbouillonculturen mit dem besten Wachsthum auf den Glycerinkartoffelbrühen ziemlich gleichen Schritt.

Wie oben schon hervorgehoben, habe ich die Züchtung der Tuberkelbacillen auf glycerinhaltigen Kartoffelbrühen bis zur dritten und vierten Generation mit vier verschiedenen Stämmen des Mikroorganismus vorgenommen, und zwar von unwesentlichen Abweichungen abgesehen, mit dem in den Versuchen $\mathrm{I}$ bis $\mathrm{V}$ veranschaulichten Erfolge. Darnach setzte das Wachsthum auf den Glycerinkartoffelbrühen in der That des Oefteren früher ein als auf Glycerinbouillon. Doch ist das keineswegs die Regel, und es lässt sich auch kein ständiger Unterschied zu Gunsten der neutralen oder der sauren Glycerinkartoffelbrühe feststellen. Bald ist das Wachsthum auf dieser, bald auf jener üppiger, bald bleibt es auf der einen, bald auf der anderen Brühe aus, und gerade dieses $\mathrm{Sch}$ wankende war charakteristisch für das Gedeihen meiner Tuberculosestämme auf den Glycerinkartoffelbrühen, wenigstens im Vergleich mit ihrem Fortkommen auf den thierischen Nährböden. Irgend welche Aenderung dieserVerhältnisse trat auch in der zweiten, dritten und vierten Generation nicht ein, ich habe im Gegentheil den Eindruck gewonnen, dass die Lebensfähigkeit der Tuberkelbacillen auf den Glycerinkartoffelbrühen eine gewisse allmähliche Einbusse erleidet. Dafür spricht einmal das regelmässige, frühzeitige Untersinken der Culturen und dann die Thatsache, dass gar nicht selten Impflinge von 
Glycerinkartoffelbrüheculturen auf den gleichen Nährböden überhaupt nicht wieder angehen.

Auch für die flüssigen Kartoffelnährböden haben sich also die von Sander berichteten Beobachtungen bei den von mir benützten Stämmen der Tuberkelbacillen und Kartoffelsorten nur ganz ausnahmsweise und auch dann nur in geringem Umfange bestätigen lassen.

Hatte Sander seine Versuche mit festen und.flüssigen, theils glycerinfreien, theils glycerinhaltigen Kartoffelnährböden angestellt, so benützte Lubinski, wie schon in der Einleitung erwähnt, eine Combination dieser Nährsubstrate mit den sonst für die Tuberculosezüchtung gebräuchlichen, nämlich Glycerinagar und Glycerinbouillon. Die Herstellung seiner Nährböden weicht von der üblichen nur insofern $a b$, als das Fleisch statt mit gewöhnlichem Wasser mit Kartoffelextract angesetzt, endlich zum Schluss nur die Hälfte des erhaltenen Gemisches neutralisirt, das übrige mit der ursprünglichen, leicht sauren Reaction verwandt wird. So erhält man vier verschiedene Nährsubstrate:

1. neutrale Glycerinkartoffelbouillon $=\mathrm{Ln}$,

2. saure Glycerinkartoffelbouillon $=\mathrm{Ls}$,

3. neutralen Glycerinkartoffelagar = Agn,

4. sauren Glycerinkartoffelagar = Ags.

Auf diesen Nährböden sollen nun die Tuberkelbacillen doppelt so schnell wachsen wie auf den sonst gebräuchlichen.

Zwecks Nachprüfung dieser Beobachtung habe ich mehrere Versuche mit zwei verschiedenen Tuberculosestämmen (Wladimiroff und Wernicke) vorgenommen. Die folgende Tabelle veranschaulicht etwa das mittlere Resultat derselben.

Versuch am 5.VI. 1898. Ausgangscultur: junge, üppig gewachsene Glycerinbouilloncultur vom 19. V. 1898.

Es wurden geimpft:

1. drei Glycerinbouillonkölbchen (I, II, III),

2. fünf neutrale Glycerinkartoffelbouillonkölbchen $\mathrm{Ln}_{1-5}$,

3. fünf saure Glycerinkartoffelbouillonkölbchen $\mathrm{Ls}_{1-5}$,

4. sieben Glycerinagarröhrchen $\mathrm{Ag}_{1-7}$,

5. zehn neutrale Glycerinkartoffelagarröhrchen $\mathrm{Agn}_{1-10}$,

6. zehn saure Glycerinkartoffelagarröhrchen Ags 1-10.

Reaction: Die neutralen Nährböden bläuen leicht rothes Lackmuspapier, blaues bleibt blau; die sauren röthen leicht blaues Lackmuspapier, rothes bleibt roth. 
W ACHSTHUM DER TUBERKELBaCILLEN.

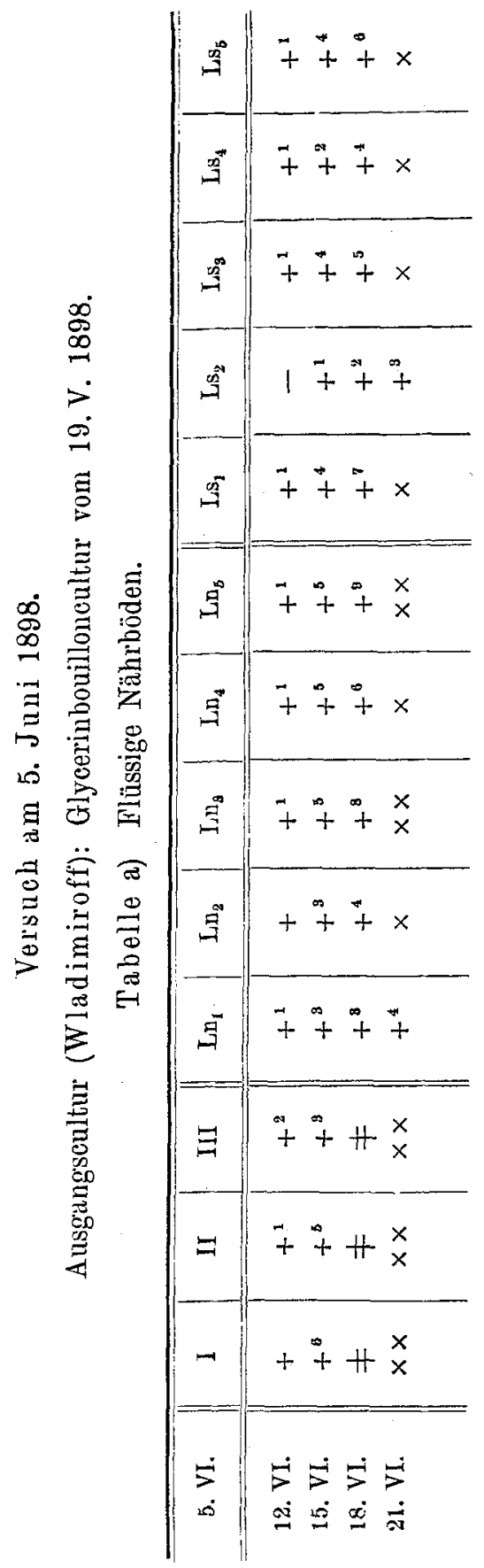

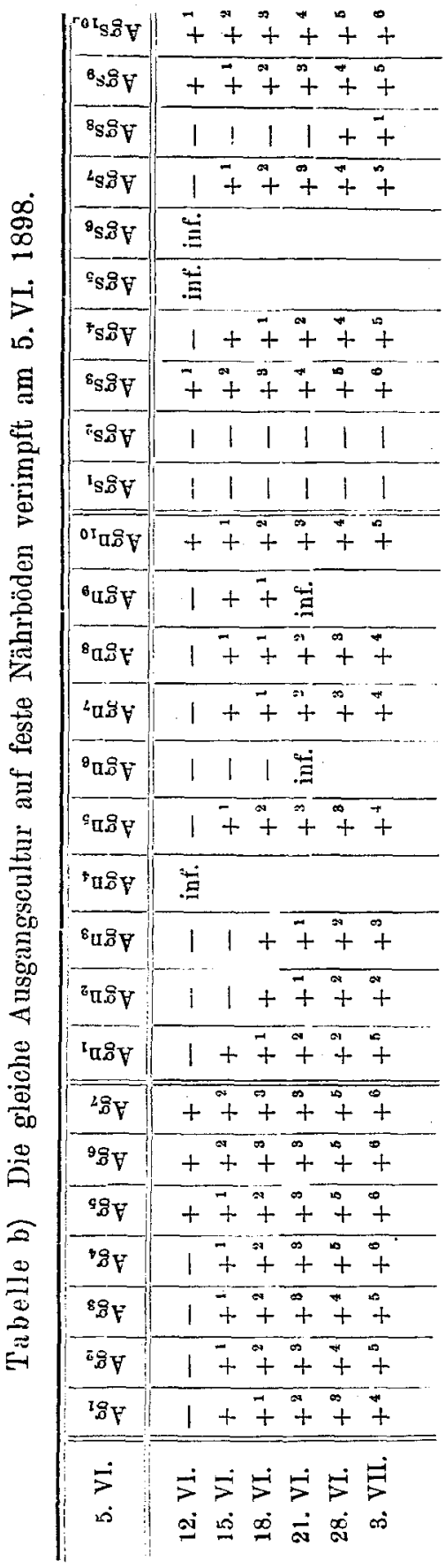


Ein Unterschied im Wachsthum auf den verschiedenen Nährböden ist kaum nachzuweisen, wenn aber vorhanden, dann eher zu Gunsten der Glycerinbouillon- und Glycerinagarculturen. Die äussere Erscheinung der Culturen auf den entsprechenden Nährsubstraten ist die gleiche; die Reaction ist am Ende des Versuches dieselbe wie zu Beginn.

Fasse ich die Ergebnisse meiner Züchtungsversuche zusammen, so sind dieselben, was die Angaben Lubinski's anlangt,y für das von mir benutzte Material - Nährsubstrat und Stammcultur - völlig negativ ausgefallen. Im Wesentlichen gilt das Gleiche für die Nachprüfung der Sander'schen Beobachtungen. Zwar ist es in einzelnen Fällen auf festen wie flüssigen, jedoch nur glycerinhaltigen Kartoffelnährböden zu einem sehr frühzeitigen und ausserordentlich üppigen Wachsthum gekommen. Indessen waren das die entschiedenen Ausnahmen, und da uns die für diese wirksamen Factoren noch völlig unbekannt sind - die Reaction spielt sicherlich keine Rolle -, so entbehren wir noch durchaus der Möglichkeit, die Ausnahme in die Regel zu verwandeln. Von einer Gewöhnung des Tuberkelbacillus an die genannten Nährböden kann jedenfalls gar nicht die Rede sein, da die Wachsthumsverhältnisse in der zweiten, dritten und vierten Generation keine Veränderung aufwiesen, ja sogar eine ungünstige Beeinflussung hervortreten liessen. Die mit Glycerin versetzten Kartoffelnährböden können dem Glycerinagar oder der Glycerinbouillon daher gewiss den Rang nicht streitig machen, den diese $\mathrm{Nähr}$ böden bisher für die Züchtung der Tuberkelbacillen eingenommen haben.

Zum Schluss ist es mir eine angenehme Pflicht, Hrn. Prof. Fraenkel für seine stete Unterstützung und seinen Rath bei dieser Arbeit meinen herzlichsten Dank auch an dieser Stelle aussprechen zu dürfen. 


\section{Litteratur-Verzeichniss.}

1. R. Koch, Mittheilungen aus dem Kaiserl. Gesundheitsamte. Bd. II.

2. Nocard et Roux, Annales de l'Institut Pasteur. 1887. Nr. 1. p. 19.

3. Pawlowski, Ebenda. 1888. Nr.6. p. 303.

4. Uschinsky, Centralblatt für Bakteriologie. Bd. XIV. Nr. 10. S. 316.

5. Kühne, Zeitschrift für Biologie. Bd. XXX. S. 239.

6. Proskauer u. Beck, Diese Zeitschrift. Bd. XVIII. S. 128.

7. C. Fraenkel, Hygienische Rundschau. 1894. Nr. 17. S. 769.

8. H. Martin, Note sur la cultur du bacille de la tuberculose. Archives de médecine expériment et d'Anatomie pathol. 1889. Nr.1. p. 77.

9. G. Sander, Ueber das Wachsthum der Tuberkelbacillen auf pflanzlichen Nährböden. Archiv für Hygiene. 1892. Bd. XVI. S. 238.

10. W. Lubinski, Zur Cultivirungsmethode, Biologie und Morphologie des Tuberkelbacillus. Centralblatt für Bakteriologie. Bd. XVIII. S. 125.

11. E. A. de Schweinitz et M. Dorset, The growth of the tuberculosis bacillus upon acid media. Bulletin Nr. 13, U. S. Departement of Agriculture. Bureau of Animal Industry. Sept. (Washington.)

12. A. Obici, Ueber den günstigen Einfluss der Luft auf die Entwickelung der Tuberkelbacillen. Centralblatt für Balteriologie. Bd. XIX. Nr.9/10. S. 314 .

13. G. Marpmann, Zur Morphologie und Biologie des Tuberkelbacillus. Ebenda. Bd. XXII. S. 582 .

14. Th. Smith, A comparative study of the bovine Tubercle bacilli and of hum bacilli from sputum. The journal of experiment. medecine. 1898. Vol. III. Nr. 4 u. 5.

15. Vagedes, Experimentelle Prüfang der Virulenz der Tuberkelbacillen. Diese Zeitsehrift. Bd. XXVIII. S. 276. 